\title{
Review of Current Distribution Measurements and Reconstruction in Cable-in-Conduit Conductors for ITER
}

\author{
Y. Ilyin and A. Nijhuis
}

\begin{abstract}
The cable-in-conduit conductors (CICC) for ITER magnets consist of more than thousand superconducting strands. Usually such cables exhibit a significant non-uniform current distribution between the strands due to inequality of strand impedances. This undesirable effect can complicate the measurement of the conductor critical transport properties, particularly for short samples. To improve the interpretation of measurement results, detailed knowledge of the current distribution is highly desired. We present a review of current unbalance measurements and current distribution reconstruction techniques in CICC as they were intensively performed during the last years. The experimental methods of current unbalance detection are described, starting with earlier attempts on sub-size CICCs and ending with more sophisticated methods on full-size conductors. The models of current identification developed by various research groups are briefly compared, emphasizing their similarities, differences and limitations. Finally, the results of current unbalance reconstructions performed by different groups on several full-size CICCs are discussed together with the implication of the results for ITER short sample conductor tests and coil performance.
\end{abstract}

Index Terms-Cable-in-conduit conductors, current distribution, Hall sensors, inverse problem, self-field measurements.

\section{INTRODUCTION}

$\mathbf{S}$ INCE the pioneering work [1] in 1995, Hall sensors (HS) and pick-up coils (PUC) were used in various experiments to detect the current redistribution process in superconducting cables, for instance [2], [3]. In the year 2000, the first measurements of the self-field profile were performed around $\mathrm{Nb}_{3} \mathrm{Sn}$ sub-size cable-in-conduit conductors (CICC) [4] aimed for the ITER conductor development program. Nowadays HSs are included in experiments as a standard tool for the reconstruction of current distribution inside the CICC, either short samples or relatively big model coils [5], [6]. Here we discuss the experimental methods, the models developed for currents identification and summarize the main results of the computations in full size ITER type CICCs. In our discussion, we focus mainly on the steady-state current unbalance caused by the non-uniform joints.

Manuscript received August 29, 2006. This work was supported by the European Communities under the contract of Association between EURATOM/FOM and was carried out within the framework of the European Fusion Development Agreement. The views and opinions expressed herein do not necessarily reflect those of the European Commission.

The authors are with the Faculty of Science and Technology, University of Twente, 7500 AE Enschede, The Netherlands (e-mail: y.ilyin@tnw.utwente.nl).

Color versions of one or more of the figures in this paper are available online at http://ieeexplore.ieee.org.

Digital Object Identifier 10.1109/TASC.2007.898163

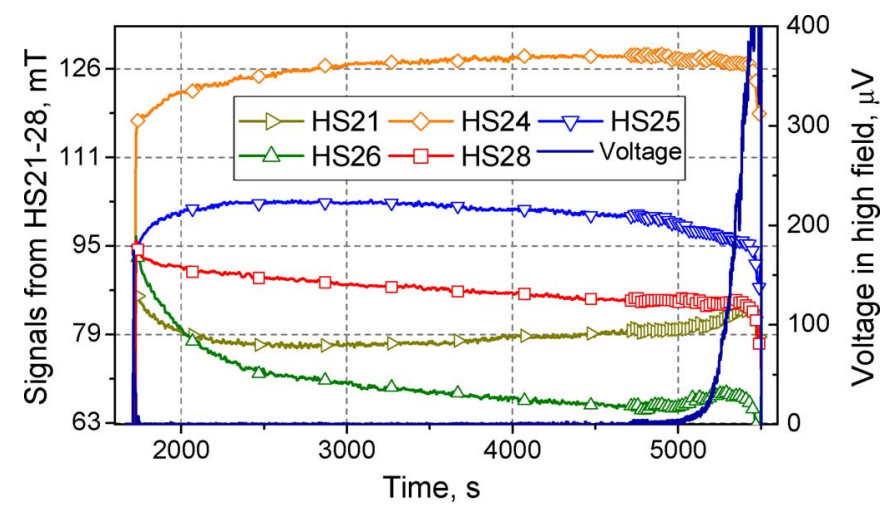

Fig. 1. Self-field changes around a $\mathrm{Nb}_{3} \mathrm{Sn}$ sub-size conductor after 3400 loading cycles. $T_{\mathrm{cs}}$ measurements at $10 \mathrm{~T}, 8 \mathrm{kA}$ and initial dI/dt $=512 \mathrm{~A} / \mathrm{s}$.

\section{EXPERIMENTS}

\section{A. Experiments on Sub-Size CICC}

First attempts to use the HSs in the experiments on sub-size CICC [4], [7]-[9] and the following effort on current reconstruction revealed the complexity of the problem. The lack of information about the experimental details related to the HS measurements, led to intolerable large errors when attempting to identify the currents in cable elements of the sub-size CICC. Therefore only qualitative conclusions could be drawn at that time based on the experimental observations.

The most appropriate experiments were the $T_{\mathrm{cs}}$ runs, in which the current is ramped up until a certain value and then kept constant, followed by raising the temperature in the conductor by a set of heaters. Observed exponential-like changes in the HS signals with time at constant current were attributed to a current redistribution between the strands [4], [7]. A typical example is shown in Fig. 1. The corresponding time constant of the transient process is in the range of $300 \mathrm{~s}$. A consequent temperature rise and voltage development in the conductor caused further change in the current distribution which was again reflected in the HS signals (see Fig. 1). The current redistribution was observed in three distant locations along the samples (in the peak field region and close to both terminations), indicating that at least a significant part of the currents redistributes through the low resistive terminations. This is also confirmed by the observed voltage changes across the terminations during the redistribution process [7].

In the $\mathrm{Nb}_{3} \mathrm{Sn}$ conductors [7] a clear change of the self-field profile with the number of loading cycles $(B \times I)$ was detected 


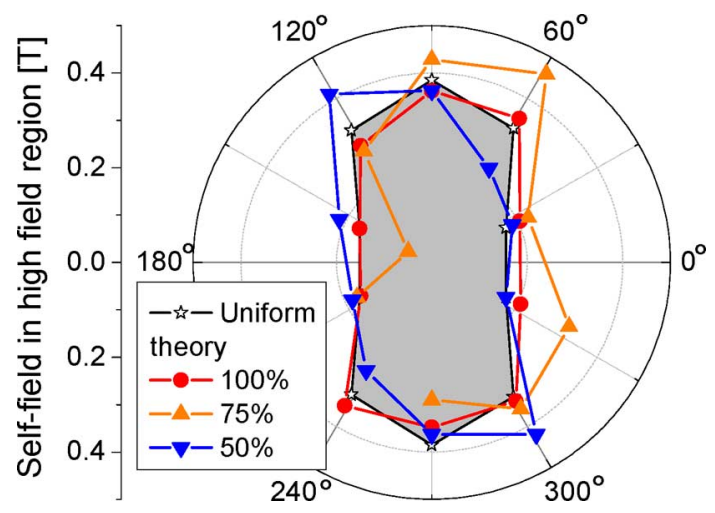

Fig. 2. Self-field profile (as a function of the angular location of the individual $\mathrm{HSs}$ ) vs. percentage of the connected strands at the terminations of a NbTi CICC. $T_{\mathrm{cs}}$ measurements at $20 \mathrm{kA}, 6 \mathrm{~T}$. The " $100 \%$ " profile is close to a uniform one. The lines connecting the points are guide to the eye.

but only in the section of the conductor in the peak field region, suggesting that the critical properties of the strands and/or their compaction have changed locally while cycling. Similar changes in the self-field profile were not observed in the NbTi conductors during cyclic loading [10].

The effect of an imposed joint non-uniformity on the current distribution in NbTi sub-size CICCs was demonstrated in [10]. For some conductors, the deviations of the self-field profile from the one corresponding to a uniform current distribution were measured even far from the terminations. An example is shown in Fig. 2. This indicates that the conductors have a long characteristic redistribution length. The critical and quench currents for varying imposed current unbalance are in good correlation with the level of current unbalance [10].

\section{B. Experiments on Full-Size CICC}

The analysis of the current distribution measurements on subsize conductors allowed one to formulate the requirements at which the current reconstruction (in absolute sense) in CICC would be possible. They can be summarized as follows:

— Use of HSs with high sensitivity $(>100 \mu \mathrm{V} / \mathrm{mT})$ and with minimized planar and quantum effects.

- Calibration of the HSs in the whole range of temperatures (i.e. $4.2 \mathrm{~K}-10 \mathrm{~K}$ ). Also desirable is a calibration in the presence of background field (up to $12 \mathrm{~T}$ ).

- Calibration of the HSs assembled in a holder, around a normal conductor (e.g. copper) having the same dimensions as the CICC.

- Knowledge of the precise position of the HS's sensitive region and orientation of the HS's plane in relation to the conduit axis.

- Knowledge of the location (radial) of the cable bundle inside the conduit (e.g. by an ultrasound technique).

- Measurements of the self-field profile around the CICC being in normal state (e.g. at $77 \mathrm{~K}$ ) representing a uniform current distribution.

- The most appropriate run for steady-state current identification is the $T_{\mathrm{CS}}$ run with a long current plateau.

All details were accounted for, to a greater or lesser extent, in the following experiments on full size ITER type CICC

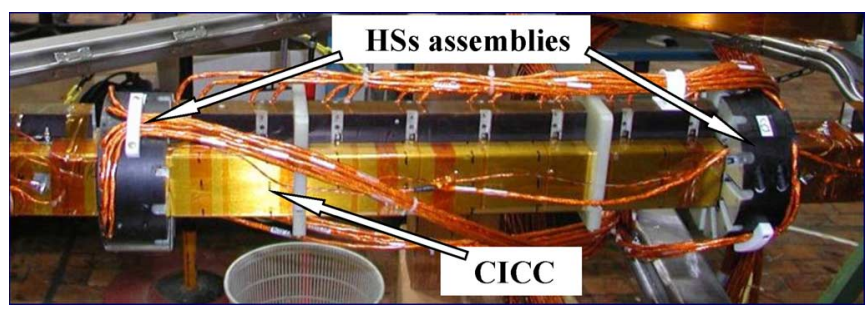

Fig. 3. HS assemblies installed on the NbTi CICC (Bus-Bar III) at FZK, Karlsruhe.

[11]-[19]. Special HS assemblies were precisely manufactured and calibrated, see for example [20]. An example view of the HS assemblies around the NbTi bus-bar is shown in Fig. 3.

An issue of the orientation of the HSs around the CICC was addressed in [21]-[24]. Several optimization models for HS positioning were proposed with the core idea to minimize the conditioning number of the coefficient matrix (see Section III), whose elements are partly functions of the HS orientation.

\section{MODELS FOR CURRENTS IDENTIFICATION}

A number of research groups developed analytical and numerical models for currents reconstruction based on the selffield measurements [11]-[24]. The unknown currents are the solution to the inverse identification problem. Assuming linear material properties with magnetic field, the governing system of linear equations can be written in a matrix form as follows:

$$
G I=B,
$$

where $\boldsymbol{B}$ is the vector of known experimental data (in general case, magnitudes of the self magnetic field and external field), $\boldsymbol{I}$ is the unknown vector of current amplitudes and $\boldsymbol{G}$ is the coefficient matrix relating the currents to the measurement data. System (1) is typically under-determined (ill posed) and has no unique solution, as the number of probes is limited $(6 \div 12)$ compared to a number of strands in the CICC (typically more than 1000). The practical limitations come from the restricted space available around the conductor and limited number of data acquisition channels.

The typical approach for the representation of the complex cable structure in the model is to consider a limited number of "equivalent" current carrying elements (i.e. groups of strands) with the unavoidable assumption of uniform current distribution within such an element. At this point the differences between the various models arise. Both the UT [8] and CEA [11], [13] models are 2D and use an assumption of 6 (or 4) straight and infinitively long petals (last minus one stage sub-cables), with a sector-like cross-section. The models from CREATE [16], THELMA [14] and CEA [19] are 3D and take into account the twist pitch of the current-carrying elements and sometimes even the shape of the coil winding. In the CREATE model the petals are introduced as helical shaped line currents, while in the THELMA and CEA model they are macro-strands with a circular-like (or sector-like) cross-section. Certainly, such approaches dramatically reduce the number of unknowns, but miss the details of current distribution inside the current elements. 
TABLE I

Steady State CuRrent Unbalance as Maximum Deviation From the AVERAge CuRrent PER Petal, IN \%

\begin{tabular}{lcccc}
\hline \hline Experiment & \multicolumn{4}{c}{ Research group } \\
& CREATE & THELMA & CEA & UT \\
\cline { 2 - 5 } CS Insert Coil & - & - & - & \pm 30 \\
PF-FSJS** $(2$ legs $)$ & - & $\pm 25 \div 50$ & $\pm 25 \div 40$ & - \\
TFMC bus-bar & \pm 25 & - & $\pm 10^{*}$ & \pm 30 \\
PFIS (2 legs) & \pm 30 & - & - & \pm 35 \\
BB-III & $\pm 20 \div 45$ & $\pm 20 \div 50$ & $\pm 20^{*}$ & $\pm 30 \div 55$ \\
TFAS $(2 \times 2$ legs $)$ & - & - & $\pm 20 \div 40$ & - \\
\hline \hline
\end{tabular}

* 4 petals model (the rest is 6 petals/macro-strands model)

** Experiment with PUC, the rest are with HSs

The unique solution of (1) can be written in most general form as:

$$
I=G^{+} B
$$

where $\boldsymbol{G}^{+}$denotes a linear operator called pseudo inverse (or Moore-Penrose inverse) of $\boldsymbol{G}$. In general, (2) is the minimumnorm, least-squares solution $\boldsymbol{I}$, that minimizes both $\|\boldsymbol{B}-\boldsymbol{G I}\|_{2}$ and $\|I\|_{2}$. In order to stabilize the solution, the SVD (singular value decomposition) technique is often utilized to calculate $\boldsymbol{G}^{+}$.

Due to the physical nature of the problem, some uncertainties in matrix $\boldsymbol{G}$ and experimental errors in vector $\boldsymbol{B}$ are unavoidable and can produce larger errors in the solution $I$. Thus, the system (1) is usually poorly conditioned and requires regularization procedures to solve. Various techniques can be considered to reduce the sensitivity of the solution to noisy data. Truncated SVD [8], [22] is a simple approach to reduce the effect of noise by neglecting singular values below a certain threshold when calculating matrix $\boldsymbol{G}^{+}$. Similarly, classical Tikhonov regularization [24] may be used to minimize the effect of noise. In any case, a regularization should be applied with care as too conservative truncation would lead to the loss of significant details in the computed current distribution.

\section{Summary of CuRREnt ReConstruction Results}

Table I summarizes the current unbalance identification results reported by different research groups in [6], [11]-[19] on various experiments. An average deviation of about $30 \%$ (mostly between 25-50\%) can be considered as a "natural" current unbalance between the six petals due to non-uniform joints for all tested ITER type conductors.

From the results in Table I it can be concluded that the more straightforward 2D models (UT and CEA) as well as more sophisticated 3D approaches (CREATE and THELMA) lead to practically the same results in terms of current unbalance.

A direct comparison between the CREATE and UT models was performed in [16] leading to the same conclusion. The limitations can be explained by the dominant role of the experimental errors and uncertainties in the identification algorithms.

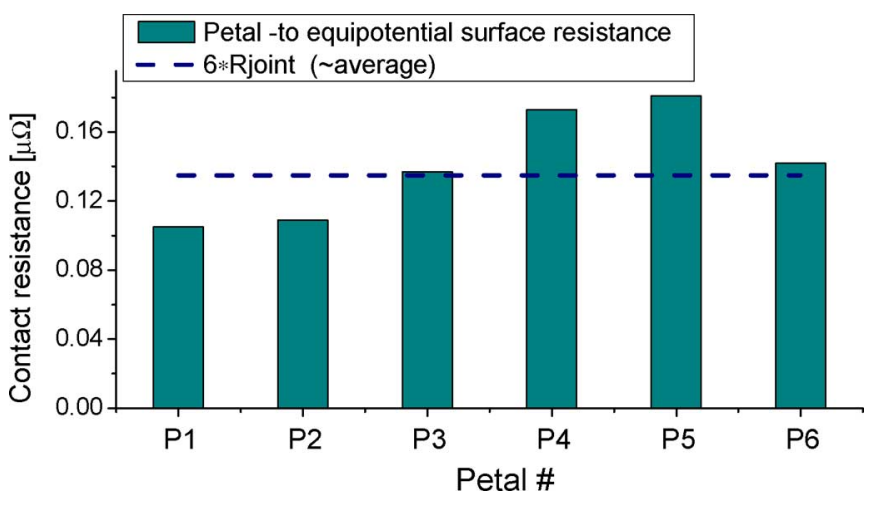

Fig. 4. Petal - to equipotential surface contact resistances in the termination of the PFIS sample as computed from the measurements in JORDI [25].

\section{COMPARISON With Direct $R_{\mathrm{c}}$ MEASUREMENTS}

The measurements of contact resistances, $R_{\mathrm{c}}$, on the PFIS joint were reported in [25]. The measurements are performed between groups of strands and the equipotential surface of the joint connecting the conductor termination and the facility current leads. Combining the results for $R_{\mathrm{c}}$ from the strand groups forming the same petal, it is possible to calculate the effective $R_{\mathrm{c}}$ between a petal and the equipotential plate. The results for six petals are presented in Fig. 4. The maximum deviation from the average $6 \cdot R_{\text {joint }}\left(\right.$ where $R_{\text {joint }}=\left(\sum_{\mathrm{i}} 1 / R_{\mathrm{i}}\right)^{-1}$ ) resistance is $\sim 25 \%$. Since in the steady state condition the currents in the petals are distributed according to the $R_{\mathrm{c}}$ distribution, the expected maximum current unbalance among six petals would be $25 \%$. This number is in a good agreement with the results in Table I, obtained by the self-field measurements on the same conductor.

\section{IMPLICATIONS FOR CONDUCTORS TESTING}

We have seen above that full size ITER type cables exhibit a steady-sate current unbalance among the six last stage sub-cables of about 30\%. In [26], [27] the impact of the current unbalance on the measured voltage-current characteristic of the PFIS sample was assessed. In [26] the simulations were performed assuming a typical SULTAN sample configuration, i.e. total length of $3.5 \mathrm{~m}$ and a $0.27 \mathrm{~m}$ distance between the bottom joint and the high field region. The values for the contact resistance between the petals outside and inside the joints were taken from the corresponding measurements. In [27] the influence of the magnetic field profile and the contact resistances in the joints was studied. It is demonstrated that the $30 \%$ current unbalance in the sample leads to a reduction of the measured critical current and $n$-value by about $10 \%$, which is significant. The situation is aggravated if the conduit outer surface in the same cross-section is not equipotential [26]. In that case the measured critical current will deviate even more from the actual critical current if averaged between all petals. It was also shown that with the realistic contact resistance values, even in a cable without the high resistive stainless steel wraps around the petals, the currents do not redistribute towards a more uniform profile until a very high voltage above $100 \mu \mathrm{V} / \mathrm{m}$ (i.e. 10 times larger 
than $I_{\mathrm{c}}$ criterion) is developed in the high field conductor section. Besides, the non-uniform current distribution causes high local voltages in the petals, eventually leading to a 'premature' quench [26].

The situation is obviously much less dramatic if the conductor has a long (compared to the characteristic redistribution length) distance between the joints and the region of peak magnetic field and/or sufficient length of the conductor being in the peak field. Although the above may seem obvious, as for example shown for the ITER Central Solenoid Coil Insert, analyzed in [28], the role of the transverse inter-petal resistance in combination with the coil geometry plays an important role as illustrated for the Poloidal Field Insert Coil in [29]. In this particular case it appeared that a higher cable inter-petal resistivity enhances the overall conductor performance by suppressing the peak local voltages.

\section{CONCLUSION}

Reconstruction of the current unbalance on the level of six last stage sub-cables of ITER conductors is possible, however the level of non-uniformity within a sub-cable can not be assessed and so the impact of this component is not accurately addressed in any of the present models. This implies a relatively large level of uncertainty, independent from the applied model. In that sense, a more detailed modeling does not necessarily lead to a higher accuracy.

It appears that the average natural degree of the current nonuniformity between the last stage sub-cables due to unequal resistivity in the joints is about $30 \%$ and mostly within $25-50 \%$. This number is confirmed by direct measurements of the joint contact resistance, where $25 \%$ deviation of petal joint resistances from the average was in good agreement with the reconstruction from the self-field profile.

\section{REFERENCES}

[1] V. S. Vysotsky, M. Takayasu, M. Ferri, J. V. Minervini, and S. S. Shen, "New method of current distribution studies for ramp rate stability of multistrand superconducting cables," IEEE Trans. Appl. Supercon., vol. 5, pp. 580-583, June 1995.

[2] N. Amemiya et al., "Current redistribution and stability of superconducting triplex cable without electrical insulation carrying non-uniform current," Cryogenics, vol. 43, pp. 249-254, March 2003.

[3] A. P. Verweij and L. Buchsbaum, "Experimental results of current distribution in Rutherford-type LHC cables," Cryogenics, vol. 40, pp. 663-670, August 2000.

[4] Y. A. Ilyin, A. Nijhuis, H. H. J. ten Kate, and P. Bruzzone, "Self field measurements by Hall sensors on the SeCRETS long sample CICCs in SULTAN," IEEE Trans. Appl. Supercon., vol. 12, pp. 1667-1671, March 2002.

[5] A. Ulbricht et al., "The ITER toroidal field model coil project," Fusion Engineering and Design, vol. 73, pp. 189-327, Oct. 2005.

[6] A. Nijhuis, Y. A. Ilyin, and H. H. J. ten Kate, "Analysis of the current distribution in the ITER CS-insert model coil conductor by self field measurements," IEEE Trans. Appl. Supercon., vol. 12, pp. 1675-1679, March 2002.

[7] Y. A. Ilyin, A. Nijhuis, H. H. J. ten Kate, B. Stepanov, and P. Bruzzone, "Self field measurements by hall sensors on the SeCRETS short sample CICC's subjected to cyclic load," IEEE Trans. Appl. Supercon., vol. 13, pp. 1752-1755, June 2003.

[8] Y. A. Ilyin and A. Nijhuis, "Case studies in reconstruction efficiency of current distribution in CICC's by self field measurements," IEEE Trans. Appl. Supercon., vol. 13, pp. 1748-1751, June 2003.
[9] A. Formisano, R. Martone, and M. Cioffi, Identification of the Current Distribution on Cable-in-Conduit Conductors From Magnetic Field Measurements Univ. of Napoli, Italy, April 2001, Technical Report.

[10] B. Stepanov et al., "Impact of impressed current unbalance on n-index," Trans. Appl. Supercon., vol. 14, pp. 1495-1498, June 2004.

[11] L. Zani, D. Ciazynski, and P. Gislon, "Current distribution between petals in PF-FSJS sample," presented at the Workshop "15 Months of CICC NbTi Results in SULTAN", Gstaad, Switzerland, Jan. 2003, unpublished.

[12] F. Bellina, "Current distribution identification in the poloidal field full-size joint sample," IEEE Trans. Appl. Supercon., vol. 16, pp. 1386-1390, June 2006.

[13] L. Zani, D. Ciazynski, R. Heller, F. Wuchner, and H. Rajainmaki, "Study of current distribution in ITER TFMC NbTi Busbar III," IEEE Trans. Appl. Supercon., vol. 16, pp. 864-867, June 2006.

[14] F. Bellina, P. L. Ribani, M. Bagnasco, L. Muzzi, E. Salpietro, L. S. Richard, and R. Zanino, "Analysis and simulation of the ITER-type NbTi bus-bar III with the THELMA Code," IEEE Trans. Appl. Supercon., vol. 16, pp. 1798-1802, June 2006.

[15] Y. A. Ilyin, A. Nijhuis, H. H. J. ten Kate, P. Bruzzone, and B. Stepanov, "Reconstruction of current unbalance in full-size ITER NbTi CICC by self-field measurements," IEEE Trans. Appl. Supercon., vol. 15, pp. 1391-1394, June 2005.

[16] A. Formisano and Y. Ilyin et al., "DC and transient current distribution analysis from self-field measurements on ITER PFIS conductor," $F u$ sion Engineering and Design, vol. 75-79, pp. 11-15, Nov. 2005.

[17] D. Ciazynski, H. Cloez, P. Decool, F. Wuechner, and L. Zani, "Current distribution measurement in the busbars of the ITER TF model coil," IEEE Trans. Appl. Supercon., vol. 13, pp. 1420-1423, June 2003.

[18] A. Formisano, R. Martone, and M. Cioffi, Interpretation of Results and Reconstruction of Current Distribution Univ. of Napoli, Italy, April 2003, Technical Report.

[19] L. Zani et al., "Analysis of DC properties and current distribution in TFAS ITER conductor samples using high $\mathrm{Jc} \mathrm{Nb}_{3} \mathrm{Sn}$ advanced strands," presented at the ASC'06 Conference, Seattle, WA, Aug. 2006, unpublished.

[20] M. Polak et al., Measuring Carriers for PFCI-FSJS Experiment Institute of Electrical Engineering, Bratislava, Slovakia, Oct. 2003, Tech. Rep..

[21] F. Bellina, P. Bettini, and F. Trevisan, "Analysis of the optimal location of magnetic field probes for the determination of the current distribution inside S/C cables," IEEE Trans. Appl. Supercon., vol. 11, pp. 2379-2382, March 2001.

[22] F. Bellina, P. Bettini, and F. Trevisan, "Optimization analyses of the magnetic measurements on multistrand SC cables," IEEE Trans. Appl. Supercon., vol. 12, pp. 1651-1654, March 2002.

[23] P. Bruzzone, A. Formisano, and R. Martone, "Optimal magnetic probes location for current analysis in multistrand superconducting cables," IEEE Trans. Magnetics, vol. 38, pp. 1057-1060, March 2002.

[24] A. Formisano and R. Martone, "Optimisation of magnetic sensors for current reconstruction," International Journal for Computation and Mathematics in Electrical and Electronic Engineering, vol. 22, pp. 535-548, 2003.

[25] P. Bruzzone, B. Stepanov, R. Dettwiler, and F. Staehli, "Test results of contact resistance distribution in $\mathrm{NbTi}_{\text {and }} \mathrm{Nb}_{3} \mathrm{Sn}$ ITER Conductor Termination," presented at the ASC'06 Conference, Seattle, Aug. 2006, unpublished.

[26] Y. Ilyin, A. Nijhuis, and H. H. J. ten Kate, "Interpretation of conduit voltage measurements on the poloidal field insert sample using the CUDI-CICC numerical code," Cryogenics, vol. 46, no. 7-8, pp. 517-529, July-August 2006.

[27] A. Nijhuis, Y. Ilyin, and H. H. J. Ten Kate, "Influence of the magnetic field profile on ITER conductor testing," Supercond. Sci. Technol., vol. 19, pp. 783-791, 2006.

[28] A. Nijhuis, H. G. Knoopers, Y. Ilyin, A. Godeke, B. Ten Haken, and H. H. J. ten Kate, "Effect of self-field and current non-uniformity on the voltage-temperature characteristic of the ITER central solenoid insert coil by numerical calculations," Cryogenics, vol. 42, pp. 469-483, Aug. 2002.

[29] A. Nijhuis, Y. Ilyin, and H. H. J. Ten Kate, "The effect of inter-bundle resistive barriers on coupling loss, current distribution and DC performance in ITER conductors," IEEE Trans. Appl. Supercon., vol. 16, pp. 868-871, June 2006. 